\title{
Simulation of Time-Reversal Processing for Electromagnetic Communication
}

\author{
G. J. Burke, A. J. Poggio
}

July 25, 2003

U.S. Department of Energy

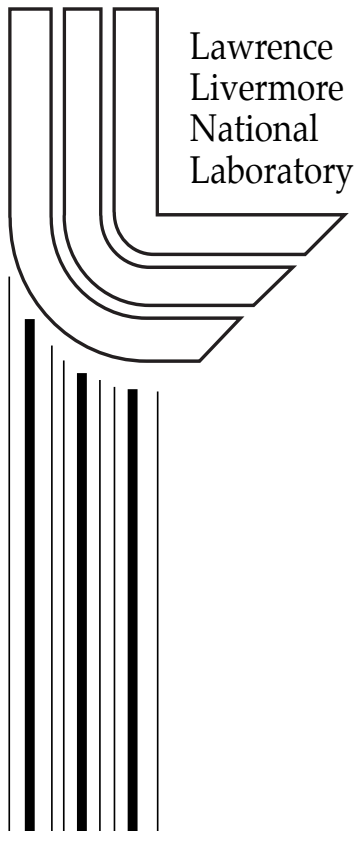




\section{DISCLAIMER}

This document was prepared as an account of work sponsored by an agency of the United States Government. Neither the United States Government nor the University of California nor any of their employees, makes any warranty, express or implied, or assumes any legal liability or responsibility for the accuracy, completeness, or usefulness of any information, apparatus, product, or process disclosed, or represents that its use would not infringe privately owned rights. Reference herein to any specific commercial product, process, or service by trade name, trademark, manufacturer, or otherwise, does not necessarily constitute or imply its endorsement, recommendation, or favoring by the United States Government or the University of California. The views and opinions of authors expressed herein do not necessarily state or reflect those of the United States Government or the University of California, and shall not be used for advertising or product endorsement purposes.

This work was performed under the auspices of the U. S. Department of Energy by the University of California, Lawrence Livermore National Laboratory under Contract No. W-7405-Eng-48.

This report has been reproduced directly from the best available copy.

Available to DOE and DOE contractors from the Office of Scientific and Technical Information P.O. Box 62, Oak Ridge, TN 37831

Prices available from (423) 576-8401

http://apollo.osti.gov/bridge/

Available to the public from the National Technical Information Service

U.S. Department of Commerce 5285 Port Royal Rd., Springfield, VA 22161 http://www.ntis.gov/

OR

Lawrence Livermore National Laboratory Technical Information Department's Digital Library http://www.llnl.gov/tid/Library.html 


\title{
Simulation of Time-Reversal Processing for Electromagnetic Communication *
}

\author{
G. J. Burke, A. J. Poggio \\ Lawrence Livermore National Laboratory, Livermore, CA 94550
}

\begin{abstract}
Time-reversal processing was simulated for several possible electromagnetic communication channels, including random point scatterers, large plates and a conducting cavity. Communication was from a single transmitting antenna to a receiving array. The effectiveness of time-reversal processing was compared for a single receiver and the array. The aim of these simulations was to determine a communication environment that would give an interesting level of multipath interference and that can be constructed in a laboratory.
\end{abstract}

\section{Introduction}

Time-reversal $(\mathrm{T} / \mathrm{R})$ processing in communication systems has been demonstrated to offer increased signal levels and channel capacity in a multi-scattering environment $[1,2,3]$. Most experimental and simulated demonstrations of this technique have been conducted for acoustic signals, since suitable transducers and receivers are available and the required frequencies, bandwidths and path lengths are reasonable for laboratory equipment.

The purpose of this work in simulating electromagnetic communication has been to examine scenarios that will yield an interesting level of multi-path interference to demonstrate T/R processing, and that can be duplicated in laboratory experiments. Significant interference in a pulse modulated signal can occur when there is inter-symbol interference due to the multiple signal paths, or equivalently a large time-delay bandwidth product. Scaling a real communication link that may extend over tens of miles down to laboratory scale would require impractically large bandwidths. However, larger time delays can be obtained when extensive multiple scattering occurs, and such a highly scattering, chaotic channel is what $T / R$ needs to perform well.

The first simulation considered was point scatterers randomly distributed in a box, where each point reflects the transmitted signal toward the receiver. The Green's function for this case can be evaluated relatively fast for a large number of scatterers. However, since multiple scattering is not allowed, the maximum time delay $\Delta t$ for scattering paths is limited by the size of the bounding box. A large $\Delta t \Delta \omega$ product can be achieved in the simulation by using a large bandwidth signal, but that would be impractical with available laboratory equipment. Results are included here to demonstrate the basic process of $\mathrm{T} / \mathrm{R}$ with a receiving array.

The next scenario considered was scattering by a number of large plates that resemble the sides of buildings in an urban environment. The scattering was modeled with the UTD code NECBSC [4] which traces rays as they are reflected and diffracted between the plates. In principle the scattering between plates allows an unlimited number of multiple bounces. However, the signal strength can decrease rapidly on diffraction, and unless there is a large number of plates, rays are quickly lost from the system. Also, NEC-BSC traces a limited number of multiple reflections and diffractions before it drops the ray.

* This work was performed under the auspices of the U. S. Department of Energy by the University of California, Lawrence Livermore National Laboratory under Contract No. W-7405-Eng-48. 
The final simulation was a source and receiver array in a conducting box. The Green's function in this case is evaluated by summing images in the walls of the box. This situation yields a large number of signal paths, with a chaotic Green's function resembling that obtained in the acoustic experiments in [3]. Similar electromagnetic experiments could be conducted in the laboratory using an available cavity.

Another simulation considered was a random array of wires, resembling the cylinders used in acoustic experiments [1]. Including all wires in a matrix solution would allow unlimited multiple bounces of the signal. However, to simulate wires, even in 2D, would require a matrix whose order is the number of wires, and would have to be solved at many frequencies. It is unclear how many wires would need to be modeled to eliminate coherent waves going around the sides. Also, building a wire array for measurements would probably be more difficult than using a cavity. So the wire array was not pursued.

Results of these numerical simulations are presented below.

\section{T/R Simulations}

For the simulations of time-reversal communication we consider an information signal $i(t)$ transmitted from a source to a receiver located at $r_{i}$ through a channel with Green's function $g\left(r_{i}, t\right)$, so that the received signal is

$$
z_{i}(t)=g\left(r_{i} ; t\right) * i(t)
$$

A pilot signal $p(t)$ is also transmitted through the channel, yielding the received pilot signal

$$
z_{p i}(t)=g\left(r_{i} ; t\right) * p(t)
$$

In $\mathrm{T} / \mathrm{R}$ processing at the receiver the received pilot is reversed and convolved with the received information signal and with the known pilot to obtain the $T / R$ received signal

$$
\begin{aligned}
R_{i}(t) & =z_{i}(t) * z_{p i}(-t) * p(t) \\
& =g\left(r_{i} ; t\right) * g\left(r_{i},-t\right) * p(t) * p(-t) * i(t)=C_{g g i}(t) * C_{p p}(t) * i(t)
\end{aligned}
$$

where $C_{g g i}(t)$ and $C_{p p}(t)$ are the autocorrelations of $g\left(r_{i} ; t\right)$ and $p(t)$. If these autocorrelations approximate impulses, then $R_{i}(t)$ should yield a good reproduction of the information signal $i(t)$.

Several forms for time-reversal receivers are discussed in [3] where the processing can be done on transmission or on reception. Since noise is not included in the simulations here, processing on transmission or reception reduce to the same result, so only the form of equation (1) will be considered.

\section{Point Scatterers}

As a simple simulation of communication in a multipath channel we evaluated scattering by $N_{s}$ randomly located points in a bounding box. The transmitter was a single antenna located at $\mathbf{r}_{s}$ and the receiver was an array of 11 elements with locations $\mathbf{p}_{i}$. The signal at receiver $i$ is the sum of the direct and scattered components

$$
G_{i}(f)=\frac{e^{-j k R_{s i}}}{R_{s i}}+\sum_{n=1}^{N_{s}} \frac{e^{-j k\left(R_{s n}+R_{n i}\right)}}{R_{s n}+R_{n i}}
$$


where $k=2 \pi f / c, R_{s i}=\left|\mathbf{p}_{i}-\mathbf{r}_{s}\right|, R_{s n}=\left|\mathbf{r}_{n}-\mathbf{r}_{s}\right|$ and $R_{n i}=\left|\mathbf{p}_{i}-\mathbf{r}_{n}\right|$. Since the scattered waves are assumed to expand spherically from the transmitter, the scatterers can be considered to be perfect mirrors aimed in turn at each receiver. The Green's function to each receiver was obtained by evaluating (2) at 8192 frequencies from 0 to $40 \mathrm{GHz}$ for each receiver location and computing the inverse Fourier transforms.

Results for a single scattering point are shown in Fig. 1. The transmitted signal is a Gaussian pulse of $0.5 \mathrm{~ns}$ full-width-half-max, shown in red in Fig. $1 \mathrm{~d}$ and e. The 11 receiving antennas are spaced by $0.4 \mathrm{~m}$, or 0.83 wavelengths at the half power frequency of the pulse spectrum. Convolution with the Green's functions yields the received signals in Fig. 1b, where the curve colors are keyed to the colors of the receiving array points. The curves in Fig. 1b between 46 and 48 ns are arriving at each receiver by the direct path, with curves for receivers 7-11 overwriting curves 1-5 due to symmetry. The curves from 50 to $60 \mathrm{~ns}$ are arriving from the scattering point.

Since the simulated Green's functions are known, the T/R process can be implemented as

$$
R_{i}(t)=g\left(r_{i} ; t\right) * g\left(r_{i},-t\right) * i(t)=C_{g g i} * i(t)
$$

with the pilot assumed to be an impulse. The result of applying $\mathrm{T} / \mathrm{R}$ on each received signal is shown in Fig. 1c. $\mathrm{T} / \mathrm{R}$ cannot really work well with a single scatterer, but it does correct the phases of the direct signals, and the reflected pulses are reduced by being squared in the autocorrelation. The result of summing the $T / R$ signals from the 11 receivers is shown in Fig. 1e along with the summation of the raw received signals. The simple sum of the received signals implements a broadside array, but it is not optimum due to the curvature of the incident wave over the array length. The received and $\mathrm{T} / \mathrm{R}$ signals for receiver 5 alone are shown in Fig. $1 \mathrm{~d}$. The $T / R$ result shows only the benefit of squaring the reflected wave.

The same set of curves is shown in Fig. 2 for 50 randomly positioned scattering points in the $5 \times 5 \mathrm{~m}$ vertical bounding box. In this case the $\mathrm{T} / \mathrm{R}$ processing does not work well and the transmitted Gaussian pulse cannot be recovered. The reason is that at low frequencies all terms in equation (2) add in phase, so that $C_{g g i}(t)$ is not impulsive. This problem would not occur if the transmission was limited to a sufficiently high frequency band. To allow $\mathrm{T} / \mathrm{R}$ to work for the low frequency Gaussian pulse, equation (2) was modified to include a random sign in the scattering terms. The receiving transfer functions become

$$
G_{i}(f)=\frac{e^{-j k R_{s i}}}{R_{s i}}+\sum_{n=1}^{N_{s}} S_{n} \frac{e^{-j k\left(R_{s n}+R_{n i}\right)}}{R_{s n}+R_{n i}}
$$

where $S_{n}$ is +1 or -1 with equal probability. With the random sign, the scatterers can be thought of as a mixture of electrically and magnetically conducting plates. The resulting curves for received signals and $T / R$ signals are shown in Fig. 3. In this case the $T / R$ array output in Fig. 3e recovers the transmitted Gaussian pulse. Simply summing the raw received signals over the array does not recover a usable signal. The results in Fig. 3d, using receiver element 5 alone, show that $\mathrm{T} / \mathrm{R}$ improves the resolution of the pulse, but not as well as the $T / R$ array.

These results demonstrate that a $\mathrm{T} / \mathrm{R}$ array can recover a transmitted signal that is highly corrupted by multipath interference. However, constructing the array of many aimed reflectors in a laboratory environment would be difficult. If non-directional scatterers are used, with the waves 
expanding spherically from the source and then spherically from each scatterer the interfering signals are reduced, and the result becomes less interesting.

\section{$\underline{\text { Scattering by Plates }}$}

For a configuration somewhat easier to construct, we next simulated a number of large parallel plates resembling sides of buildings in an urban environment. The plates were distributed randomly, but a particular configuration of eight plates, shown in Fig. 4, was used for most simulation experiments. The nine receiving antennas are spaced by $0.25 \mathrm{~m}$, from -1 to $1 \mathrm{~m}$ in the transverse dimension. The propagation through the plates was modeled with the UTD code NEC-BSC [4] which traces rays through reflections, edge diffractions and shadowing by the plates. NEC-BSC was configured to include rays with single or double reflections, reflection-diffraction or diffractionreflection, but dropped rays with more than two reflections or more than one diffraction. This limitation did not appear important here, since higher order reflections were unlikely to occur, and rays with more diffractions would be weak compared to more direct rays. The rays to receiver 8 with strengths greater than $-40 \mathrm{~dB}$ below the direct ray are shown in Fig. 4, where the color indicates the power density at the final point of the ray segment. When rays reflect the color changes only by the spreading factor to the end of the reflected ray segment, while on diffraction there is a larger drop in power density.

The Green's functions for propagation through the plates were obtained by running the NECBSC model at 4094 frequencies from 0.005 to $20.47 \mathrm{GHz}$. The UTD solution is not accurate for the first few frequencies of this range, so extrapolation was used from 0 to $60 \mathrm{MHz}$ with appropriate phase change included. The Green's functions were then obtained from inverse Fourier transforms. The transfer functions and Green's functions are shown in Fig. 5. The Green's functions show delayed responses for the various reflected and diffracted rays that differ considerably from one receiver to another.

A number of T/R simulations were run with these Green's functions, including transmission of a Gaussian pulse or pulse coded signals extending to low frequency. As a more realistic case, a pulse coded signal was modulated on a $5 \mathrm{GHz}$ carrier. The basic pulse width was 1 ns for a bandwidth of about $1 \mathrm{GHz}$. The pulse signal was a repeating pattern of a narrow pulse and a wide pulse. The signal and the spectrum of the modulated carrier are shown in Fig. 6. The Green's functions were each convolved with this pulse signal to get the raw received signals at each receiving antenna. The results for receivers 4 and 5 are shown in Fig. 7, along with the detector and threshold output. Receiver 4 was one of the best for quality of the raw signal, and receiver 5 was one of the worst. The signals after $\mathrm{T} / \mathrm{R}$ processing are shown in Fig. 8. Receiver 4 signal has been improved further, while a recognizable pulse signal is beginning to emerge from receiver 5. The results of summing the raw received signals and the $\mathrm{T} / \mathrm{R}$ results for the nine receivers are shown in Fig. 9. Both results recover the transmitted pulse signal, although the summed T/R signals would allow a wider range of threshold level.

To simulate a process closer to what could be measured in a laboratory, the $5 \mathrm{GHz}$ modulated signals were converted down to $2 \mathrm{GHz}$ by mixing with a $3 \mathrm{GHz}$ local oscillator. A 4 to $6 \mathrm{GHz}$ chirp signal was used as a pilot rather than the impulse pilot implied in the previous results. The receiver block diagram for this case is shown in Fig. 10. The chirp pilot and its autocorrelation are shown in Fig. 11. For the T/R processing of equation (1) to succeed the autocorrelation of the pilot must resemble an impulse. The autocorrelation in Fig. 11 does not look like an impulse, but the comparison with an actual impulse filtered to 4-6 GHz shows that it appears impulsive to 
a band limited signal. The sums of the raw received signals and $\mathrm{T} / \mathrm{R}$ signals after conversion to 2 $\mathrm{GHz}$ are shown in Fig. 12. The processing is essentially as effective as when $\mathrm{T} / \mathrm{R}$ is done directly at the carrier frequency of $5 \mathrm{GHz}$. Plots of the symbol error versus threshold level are shown in Fig. 13 for $\mathrm{T} / \mathrm{R}$ processing and detection done at the carrier frequency with an impulse pilot (Fig. 9) and after conversion to $2 \mathrm{GHz}$ with a chirp pilot (Fig. 12). The two processing methods yield nearly the same result, but the curves for $T / R$ have a considerably wider zero-error range than those for the direct sum of the array elements.

It should be noted that at $5 \mathrm{GHz}$ the spacing of the receiving array elements is about 4 wavelengths, so as an array it would have a narrow beam with a number of grating lobes. That might be $\mathrm{OK}$ in this case, since the grating lobes would be unlikely to find their way back to the source. However, it is probably more reasonable to consider these as separate receivers. In [1] $\mathrm{T} / \mathrm{R}$ is used to focus on separate receiver elements as independent channels. In the simulation here a single signal is transmitted, and the receivers that see more interference tend to have a weaker signal strength, and are suppressed in the summation.

\section{Communication in a Conducting Cavity}

The final simulation conducted was communication from a single source to a receiving array in a conducting cavity. The configuration is shown in Fig. 14. Transfer functions between source and each receiver were evaluated by summing images in the walls for 16000 frequencies from 0 to $5 \mathrm{GHz}$. One hundred images were summed in $x, y$, and $z$ directions for a total of $10^{6}$ images. The transfer functions to receiver element 5 are shown in Fig. 15a and b. The ideal cavity resonances are marked in Fig. 15a. They match the peaks in the numerical sum, although some resonances are not strongly excited. The smallest ripples in Fig. 15a are the result of truncating the summation at a finite number of images. A convergence acceleration technique could have been used to approximate the infinite sum, but was not felt to be necessary for the simulation. The Green's functions were then obtained from an inverse Fourier transform of the transfer functions. The autocorrelation of the Green's function is shown in Fig. 15d, and has an impulsive form.

The Green's functions for the cavity were convolved with the pulse signal of Fig. 6 on a $5 \mathrm{GHz}$ carrier to obtain the raw received signals. The received signals for elements 5 and 7 are shown in Fig. 16. The transmitted pulse signal is not recognizable in any of the raw received signals due to the long duration and chaotic nature of the Green's function. The results of T/R processing on elements 5 and 7 are shown in Fig. 17. The pulse signal is now easily recognizable. With a threshold level of 0.3 of the maximum the signal is recovered without error for receiver 7 and with one error for receiver 5 over the time interval shown. The symbol error versus threshold level for the $T / R$ signals from each of the receivers is plotted in Fig. 18. It shows considerable variation between receivers, with some having a range of zero error while others do not. Summing the 9 $\mathrm{T} / \mathrm{R}$ signals, in Fig. 19, yields an error free output, while summing the direct received signals does not give an usable result. The symbol error versus threshold level for the sum of $9 \mathrm{~T} / \mathrm{R}$ signals is shown in Fig. 21.

In Fig. 20 the basic pulse width in Fig. 6 has been scaled from 1 ns to 10 ns. The irregularity or "noise" level in the $\mathrm{T} / \mathrm{R}$ result is seen to grow due to the supperposition of many tails of the autocorrelation function $C_{g g i}(t)$ in equation (3). The number of times that the autocorrelation in Fig. $15 \mathrm{~d}$ is superimposed in the convolution with a pulse is the pulse width divided by the sample interval. The variance of the result grows with this number, and hence with the width of the pulse. This may correspond to observations that $\mathrm{T} / \mathrm{R}$ works best with a narrow band signal. The 
symbol error versus threshold level plots for the 9 receiver T/R output are compared in Fig. 21 for the $1 \mathrm{~ns}$ and $10 \mathrm{~ns}$ pulse widths. The $10 \mathrm{~ns}$ pulse is seen to result in a considerably narrower region of zero error.

\section{Conclusions}

$\mathrm{T} / \mathrm{R}$ processing in EM communications has been simulated for environments of multiple point scatterers, large plates and a conducting cavity. It has been seen that $\mathrm{T} / \mathrm{R}$ can substantially improve the resolution of the received signal in these multipath environments. $T / R$ processing with a receiving array yielded better results than with a single receiver. In the case of the conducting plates, a receiving array with electrically large element spacing gave better reproduction of the pulse signal than a narrower array without grating lobes. Complete time reversal would require capturing the waves scattered in all directions, reversing them and sending them back in the direction that they came from. Hence, ideally you need a good array structure wrapped around the entire scattering region. With a limited number of array elements there is a tradeoff between having a well designed array and capturing the scattered signal over a larger region.

The last case of communication in a cavity gave a high level of multipath interference that was mitigated by T/R processing. Suitable cavities may be available for laboratory measurements. Cavities that are not rectangular might not be amenable to modeling to high frequency, but they would be expected to yield results similar to those simulated here for a rectangular cavity.

\section{References}

[1] A. Derode, A. Tourin, J. de Rosny, M. Tanter, S. Yon, M. Fink, "Taking Advantage of Multiple Scattering to Communicate with Time-Reversal Antennas," Phys. Rev. Letters, Vol. 90, No. 1, art. 014301, Jan. 10, 2003.

[2] S. E. Skipetrov, "Information transfer through disordered media by diffuse waves," Phys. Rev. E, Vol. 67, Art. 036621, 2003.

[3] J. V. Candy, A. W. Meyer, A. J. Poggio and B. L. Guidry, Time-Reversal Processing for an Acoustic Communications Experiment in a Hostile Reverberant Environment, UCRL-JC154219, Lawrence Livermore National Laboratory, CA 2003.

[4] R. J. Marhefka, Numerical Electromagnetics Code-Basic Scattering Code (NEC-BSC Version 4.2), The Ohio State University, Columbus, OH, June 2002. 
a)

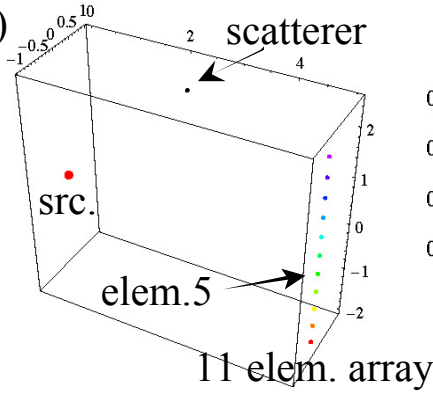

d)

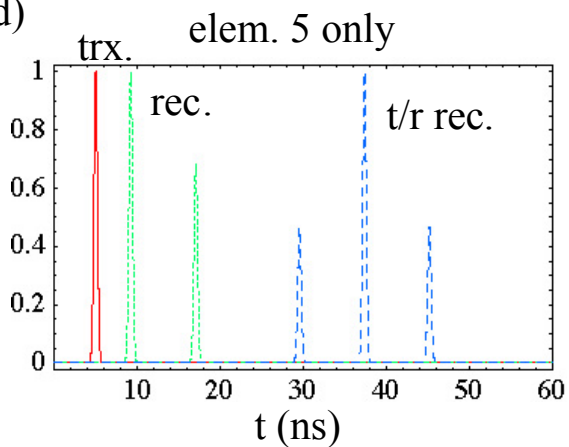

b)

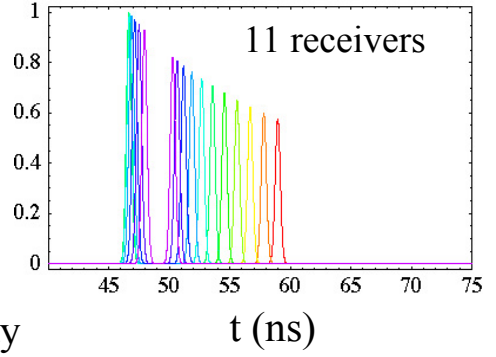

c)

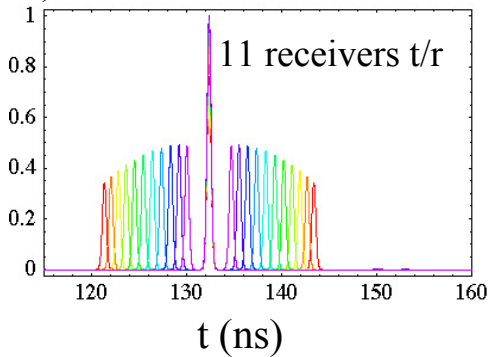

e) 11 element array

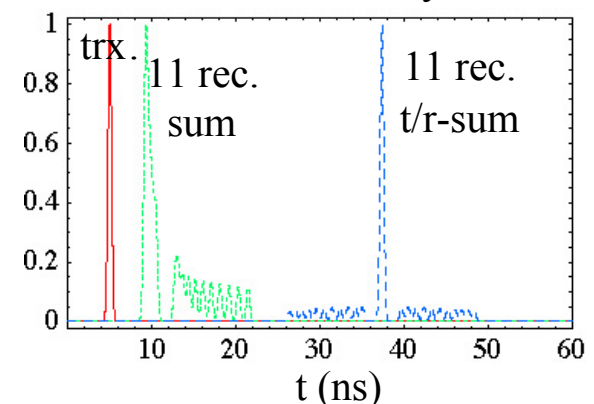

Fig. 1 Eleven element array in a box with one scatterer: a) receiver array, source and scatterer in a vertical plane, b) raw received signals for a transmitted Gaussian pulse (color keyed to receivers), c) received signals after $T / R$ processing, d) transmitted Gaussian pulse, raw received and $\mathrm{T} / \mathrm{R}$ received signals for receiver 5 only, e) transmitted Gaussian, sum of raw received signals and sum of $\mathrm{T} / \mathrm{R}$ signals for the 11 element array.

a)

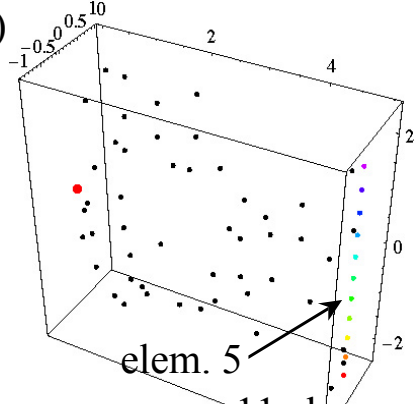

11 elem. array

d)

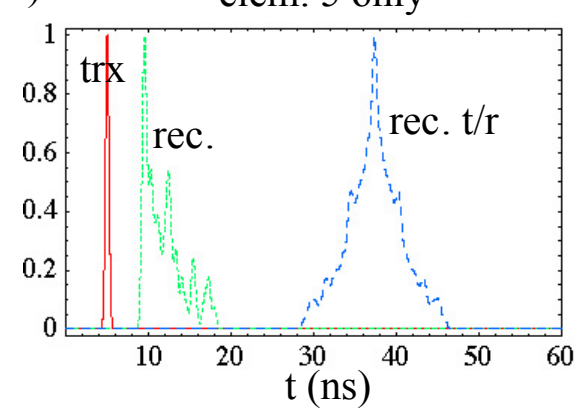

c)
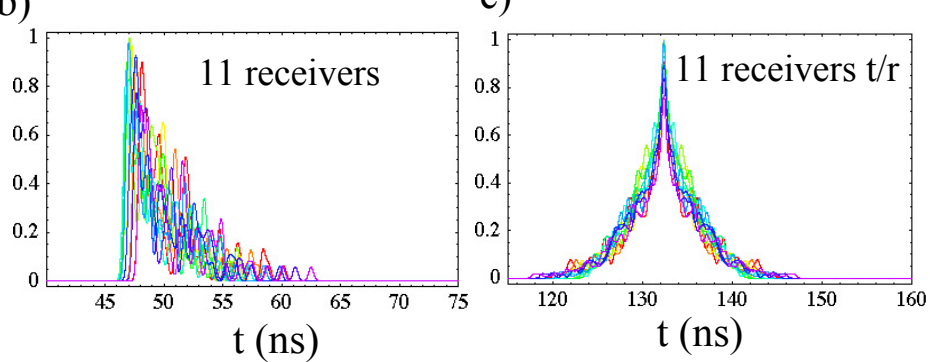

11 elem array

e)

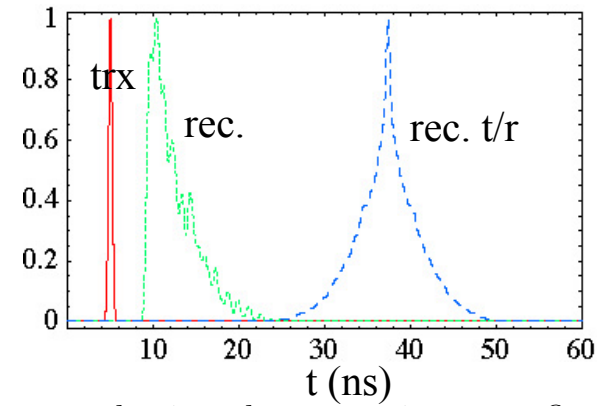

Fig. 2 Eleven element array in a box with 50 random scatterers having the same sign on reflection. Curves correspond to those in Fig. 1. 
a)

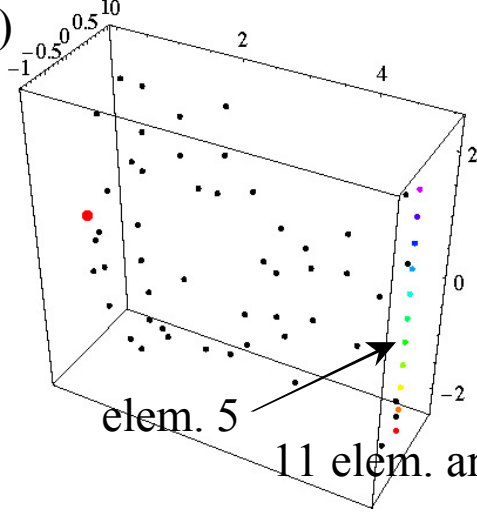

d)

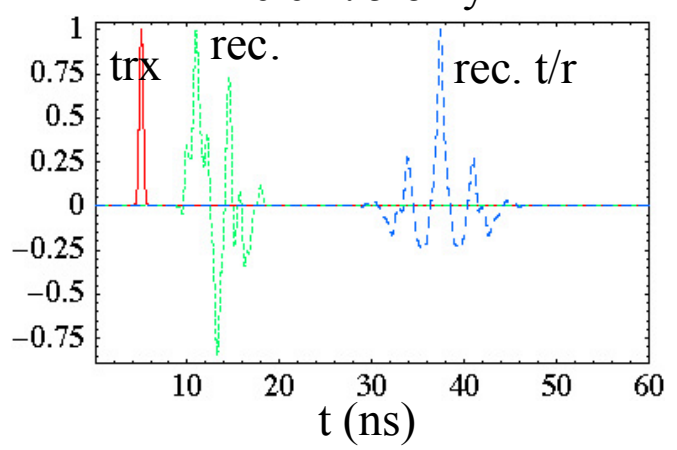

b)

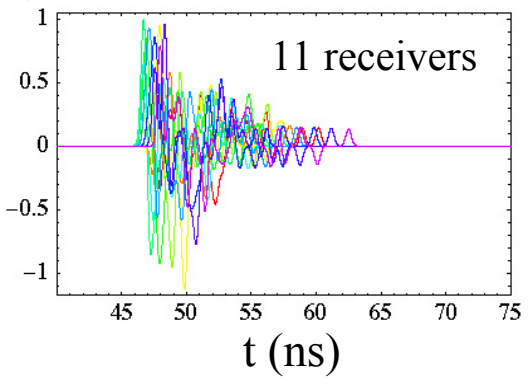

c)

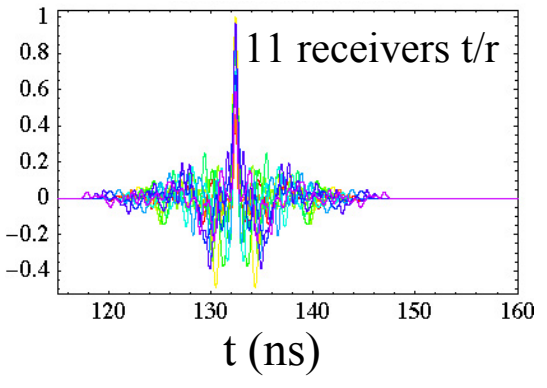

e) 11 element array

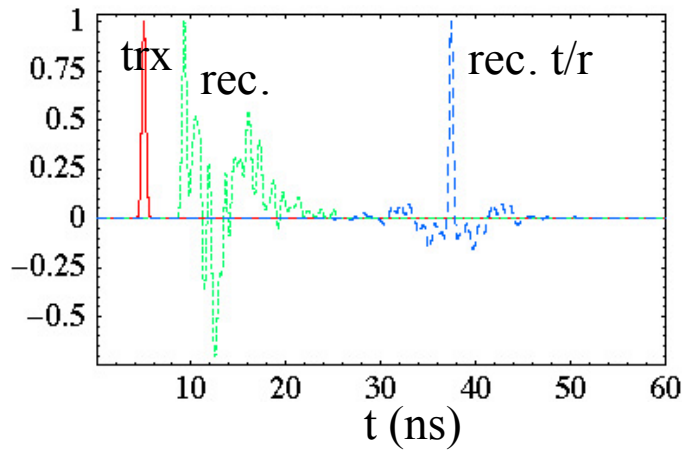

Fig. 3 Eleven element array in a box with 50 scatterers having random sign on reflection. Curves correspond to those in Fig. 1.

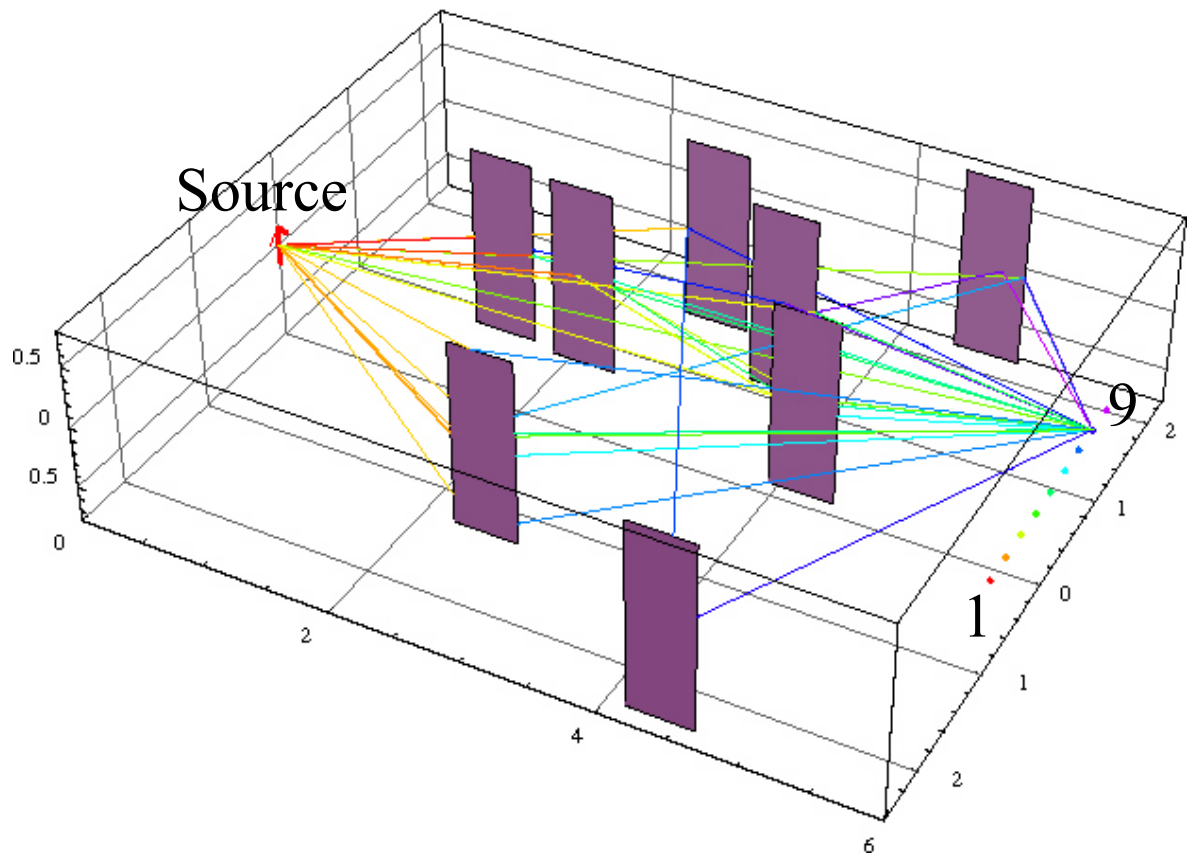

Fig. 4 Scattering by 8 plates in a box with a 9-element receiver array. Rays to $-40 \mathrm{~dB}$ below the direct ray are shown by lines with color indicating power density. 

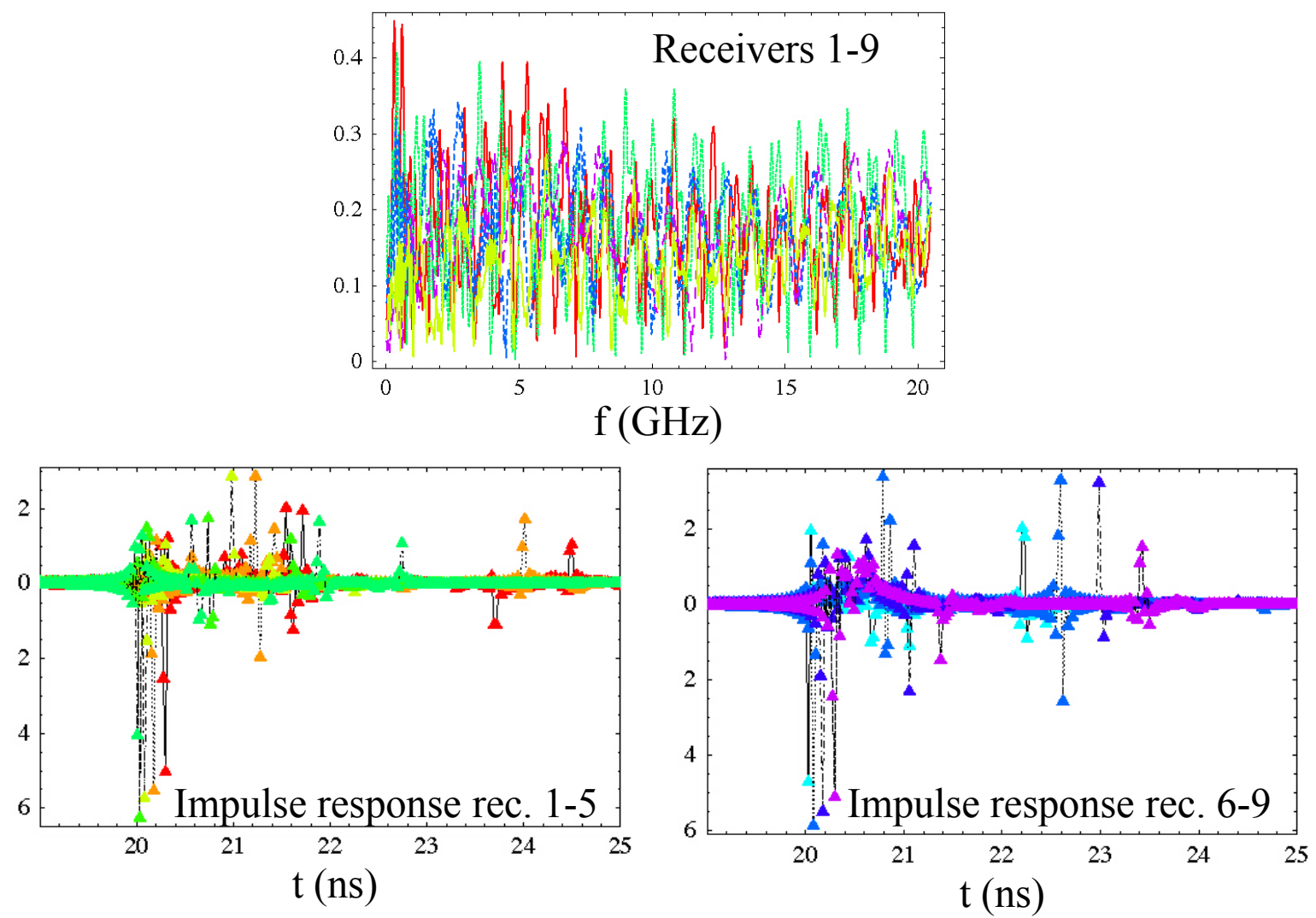

Fig. 5 Spectrum and impulse response (Green's functions) between the transmitter and the 9 receiver elements in Fig. 4. Curves are keyed to the array element colors.
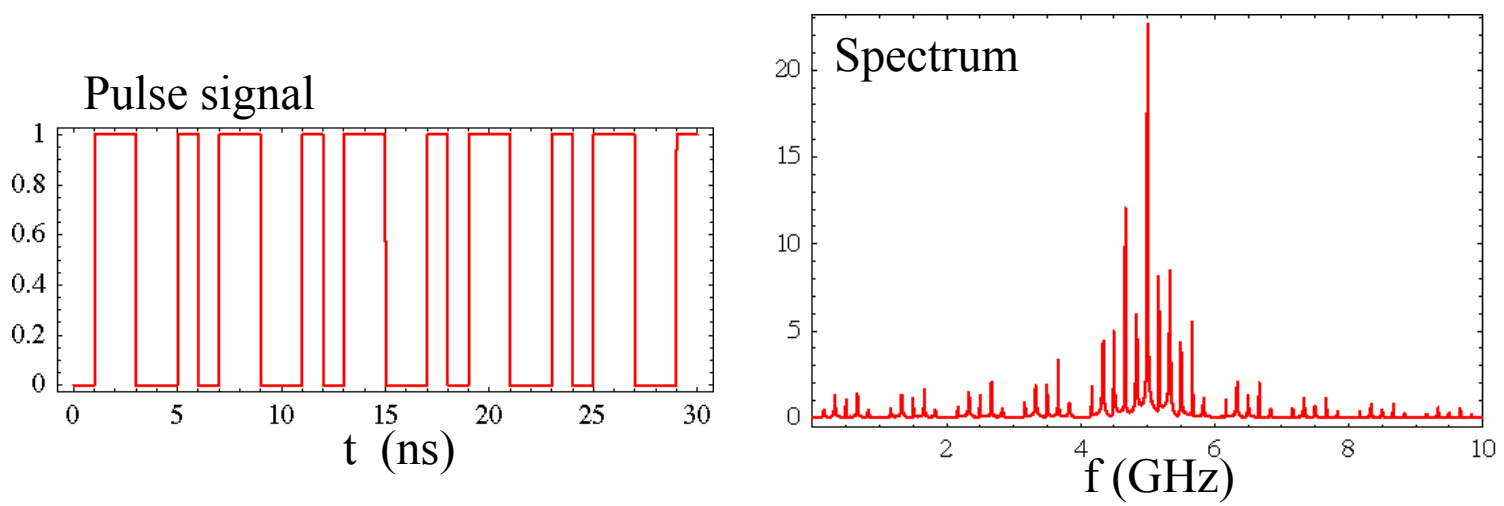

Fig. 6 Transmitted pulse signal and spectrum of the modulated 5 GHz carrier. 

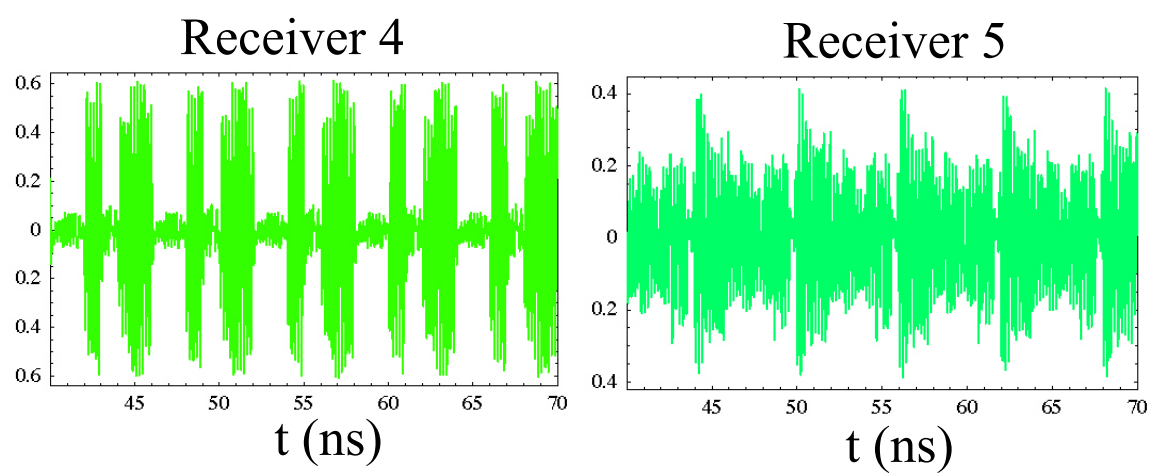

Detected and clipped
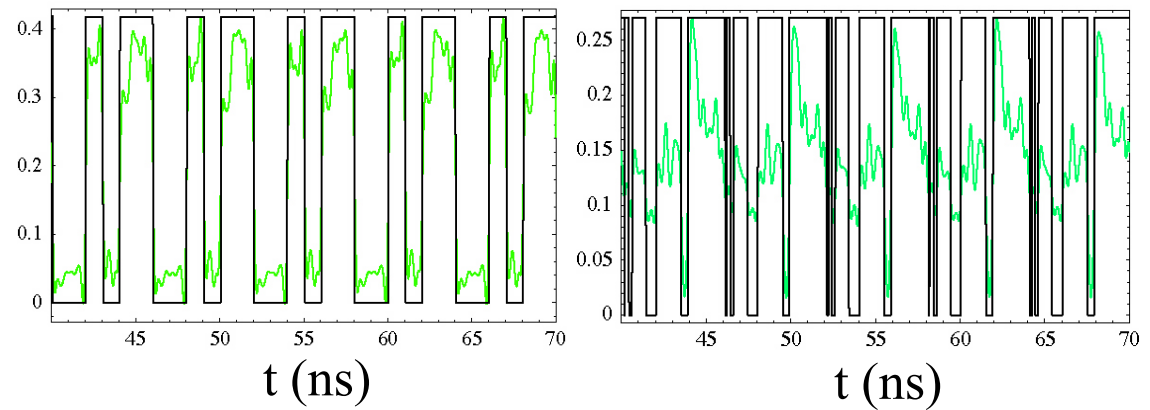

Fig. 7 Raw signal received on $5 \mathrm{GHz}$ carrier (top), and detected signal and threshold output (bottom) for receivers 4 and 5 in Fig. 4. Receiver 4 was one of the best for raw received signal, and 5 was one of the worst.
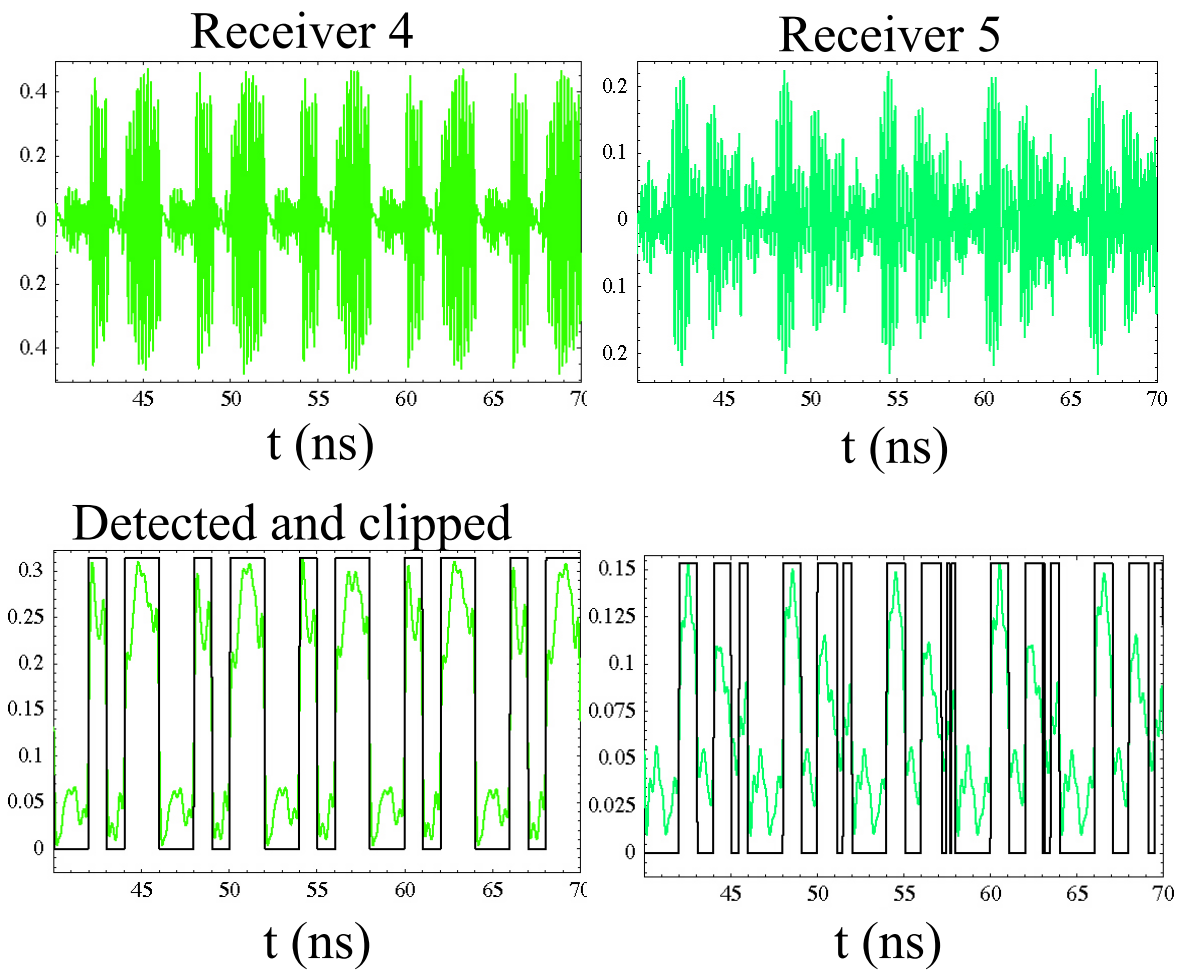

Fig. 8 Received signals from receivers 4 and 5 after T/R, and resulting detector and threshold output. 

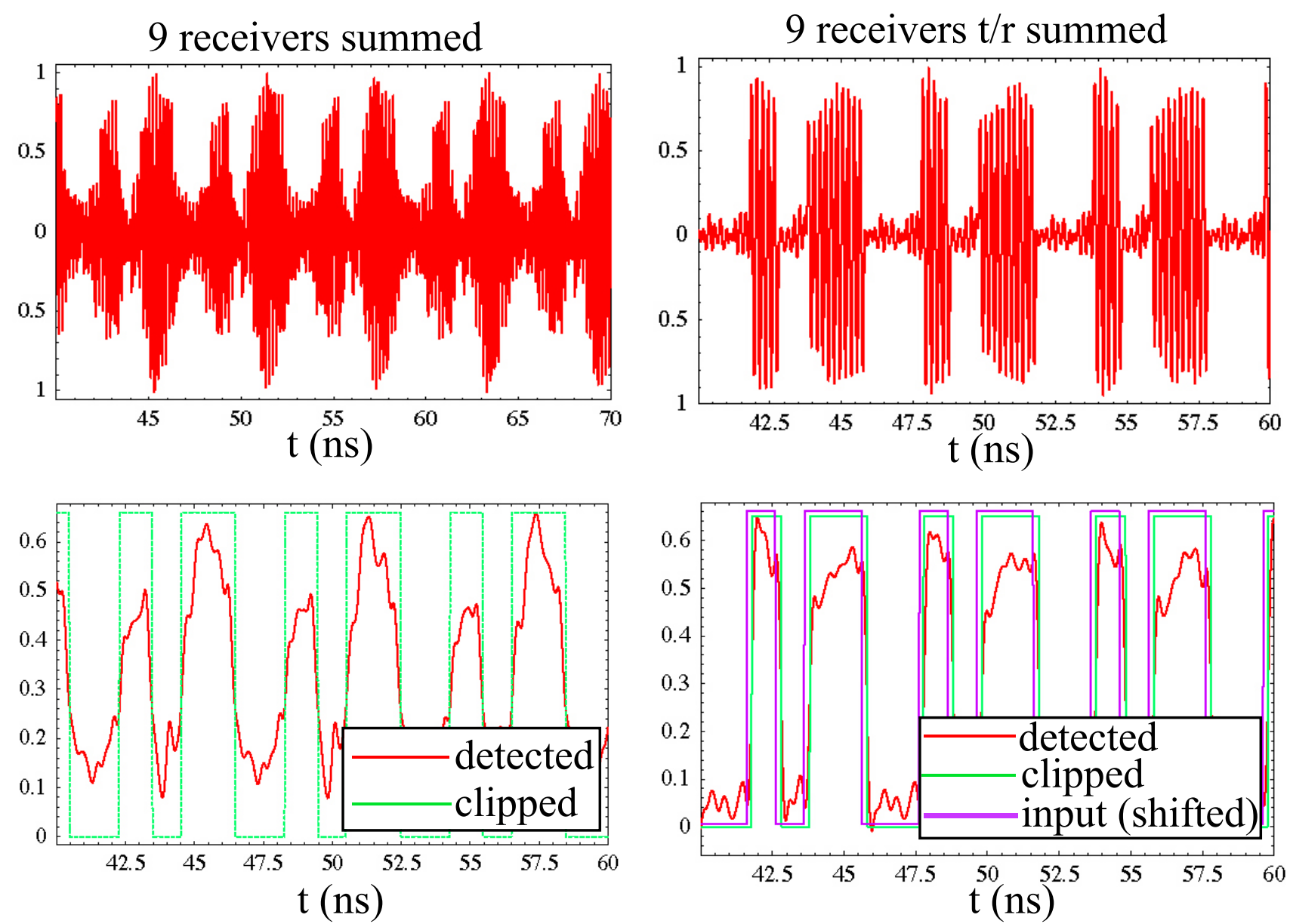

Fig. 9 Received signal from a sum of the 9 array elements in Fig. 4 and from a sum of the 9 T/R signals. 


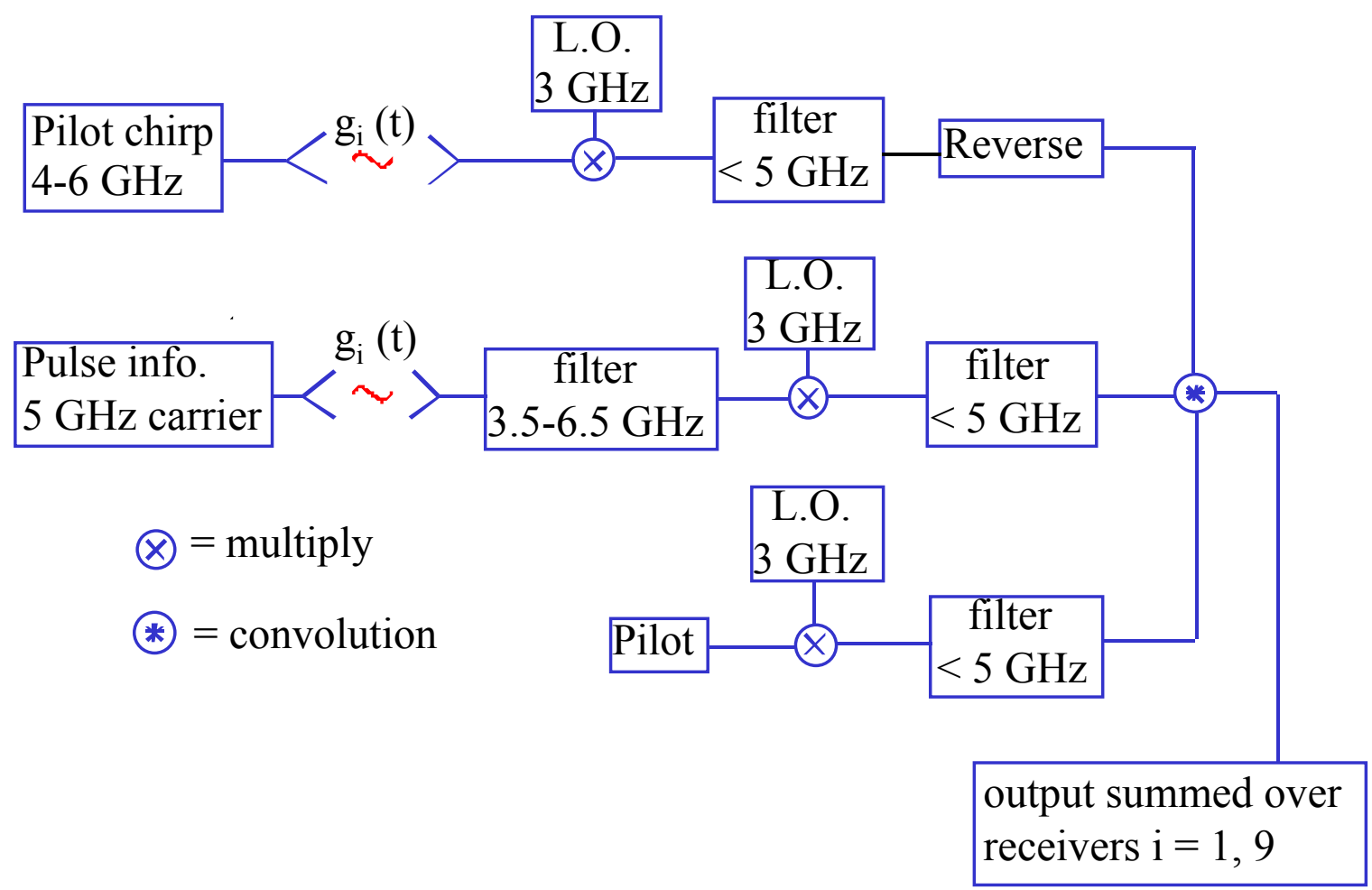

Fig. 10 Block diagram of the receiver for a $5 \mathrm{GHz}$ information signal and 4-6 GHz chirp pilot with conversion down to $2 \mathrm{GHz}$ before T/R processing. The T/R outputs are summed over the 9 receivers.

a)

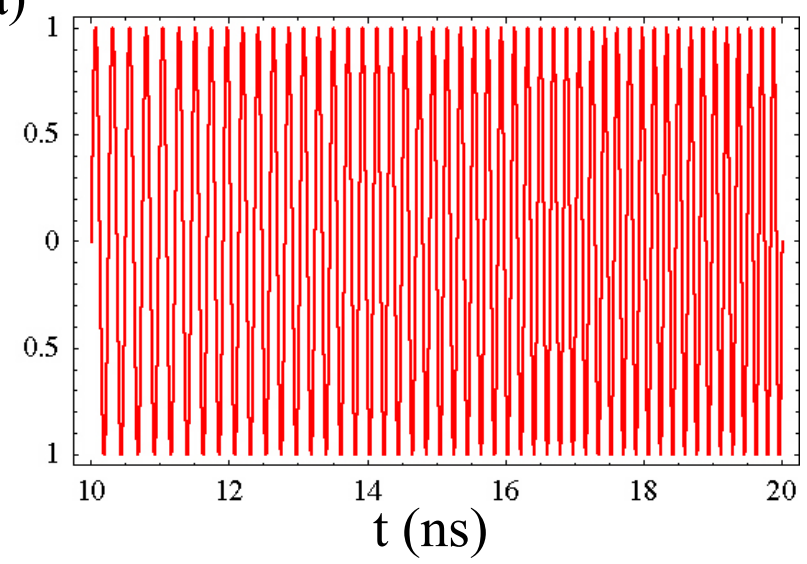

b)

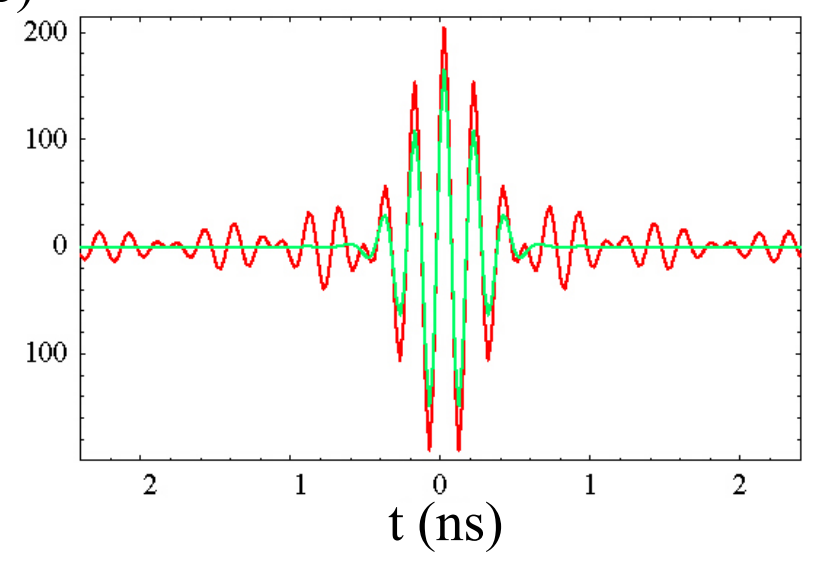

Fig. 11 a) Chirp signal, 4-6 GHz. b) Autocorrelation of the chirp (red) and an impulse filtered to 4-6 GHz (green). 

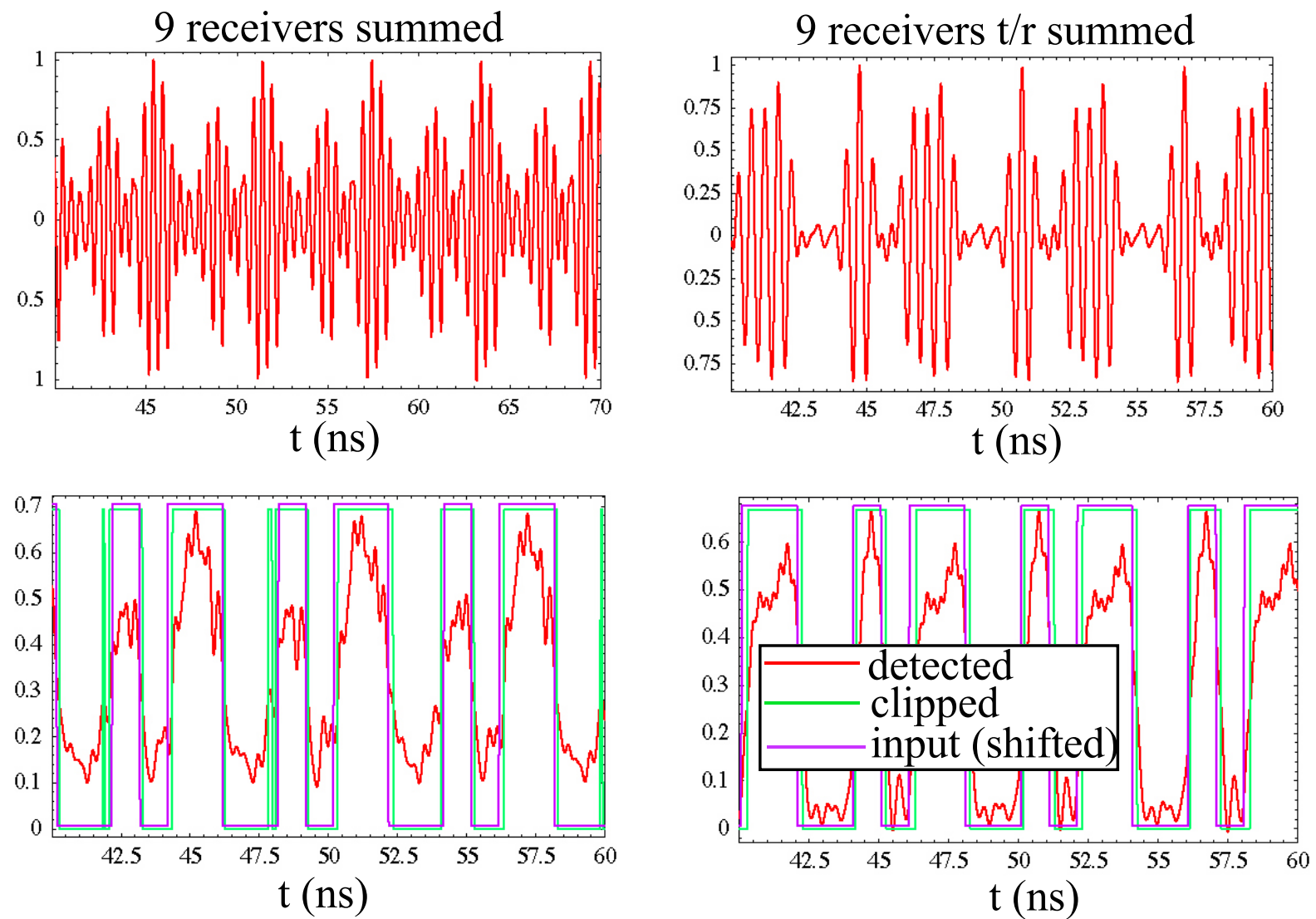

Fig. 12 Output of the receiver in Fig. 10 for the pulse signal of Fig. 6, showing the sum of raw received signals and the sum of $\mathrm{T} / \mathrm{R}$ signals over the 9 array elements. The top figures are after conversion down from 5 to $2 \mathrm{GHz}$, and the bottom figures are the detector and threshold output.

a)

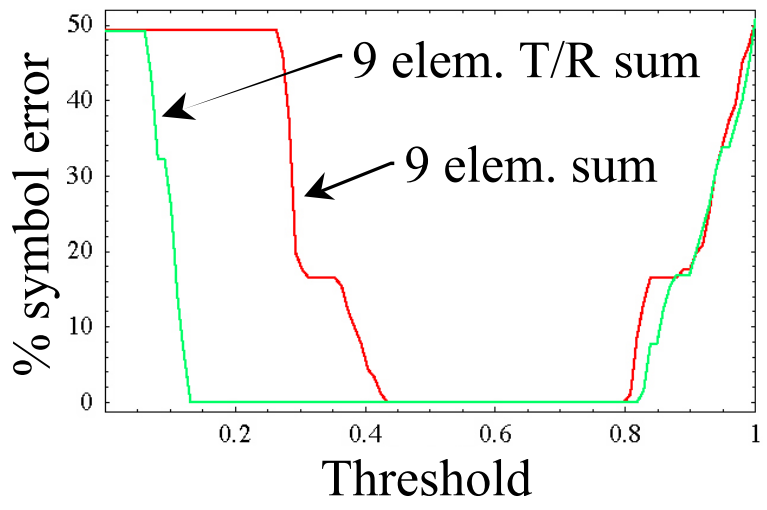

b)

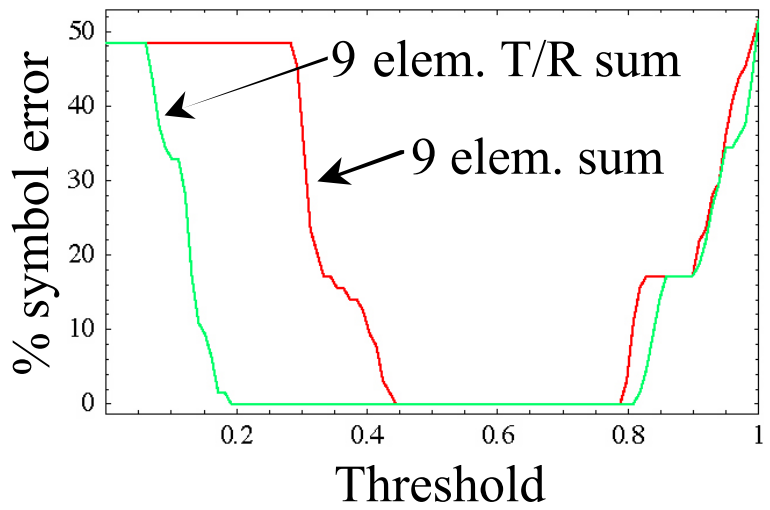

Fig. 13 Symbol error versus threshold level for the direct sum of 9 receiver elements and the T/R sum of the elements. a) T/R processing and detection done at the modulation frequency of $5 \mathrm{MHZ}$ with an impulse pilot; b) $\mathrm{T} / \mathrm{R}$ processing and detection done after conversion down to $2 \mathrm{GHz}$ with a chirp pilot. 


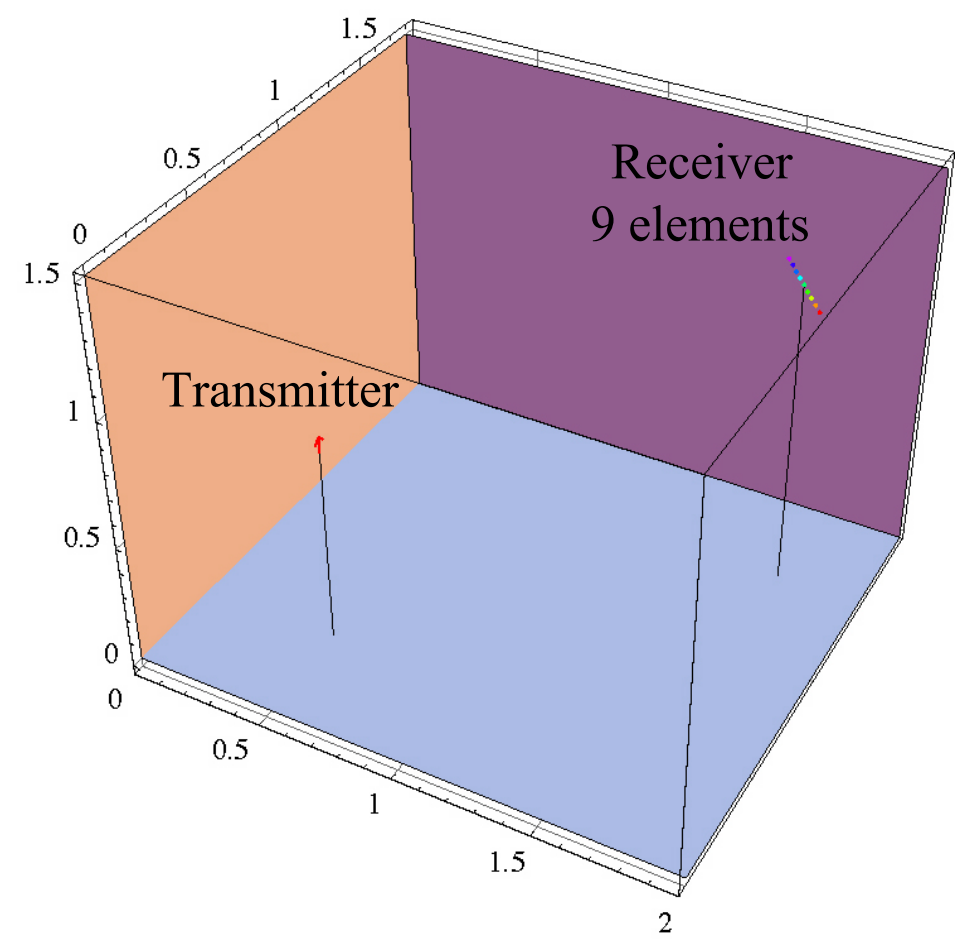

Fig. 14 Array of 9 receiver elements in a cavity with dimensions $2 \times 1.8 \times 1.5 \mathrm{~m}$. The transmitter is located at $(0.4,0.5,0.8) \mathrm{m}$. The receiving array is centered at $(1.7,1.4,1.2) \mathrm{m}$ with element spacing of $0.04 \mathrm{~m}$, and oriented normal to the direction to the transmitter.

a)
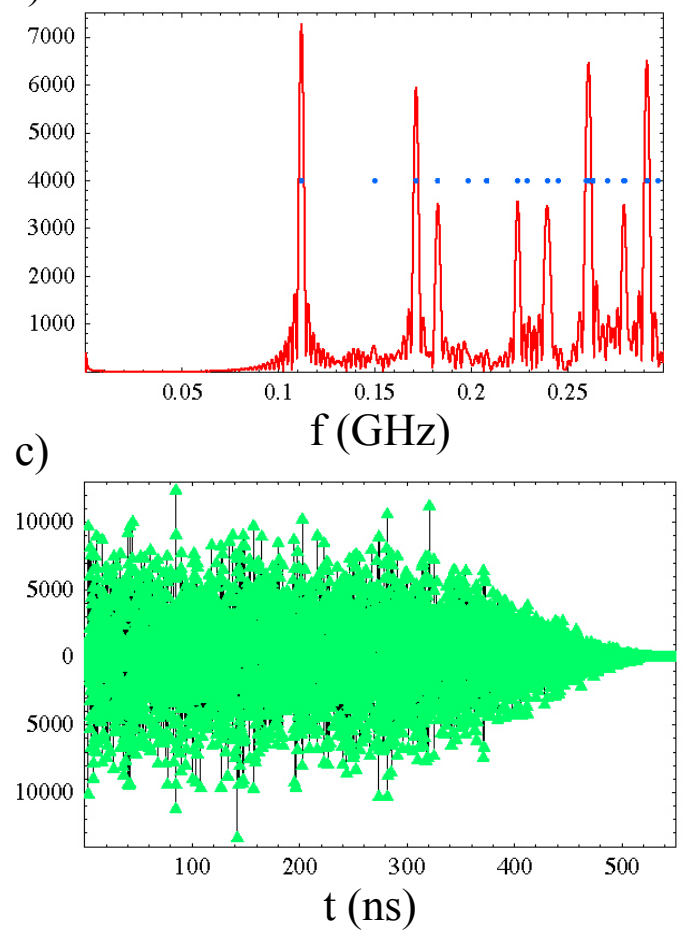

b)

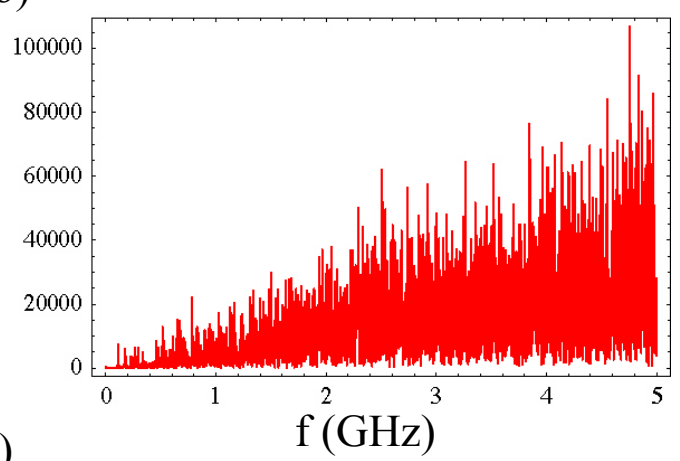

d)

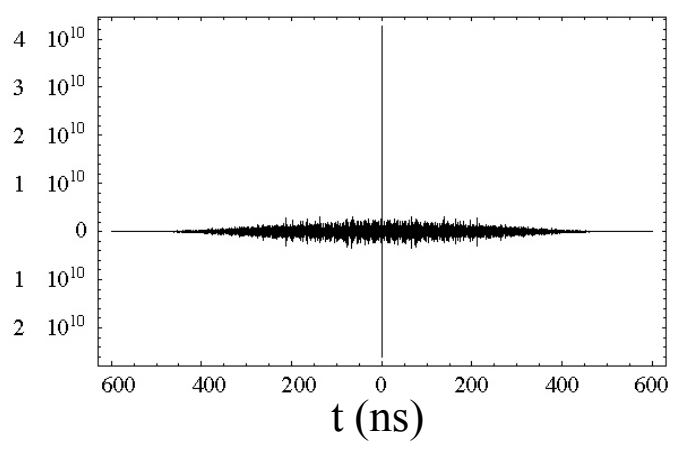

Fig. 15 a) Transfer function to receiver 5 in the cavity of Fig. 14 for low frequencies. Points indicate the ideal cavity resonances. b) Full transfer function. c) Green's function. c) Autocorrelation of the Green's function. 

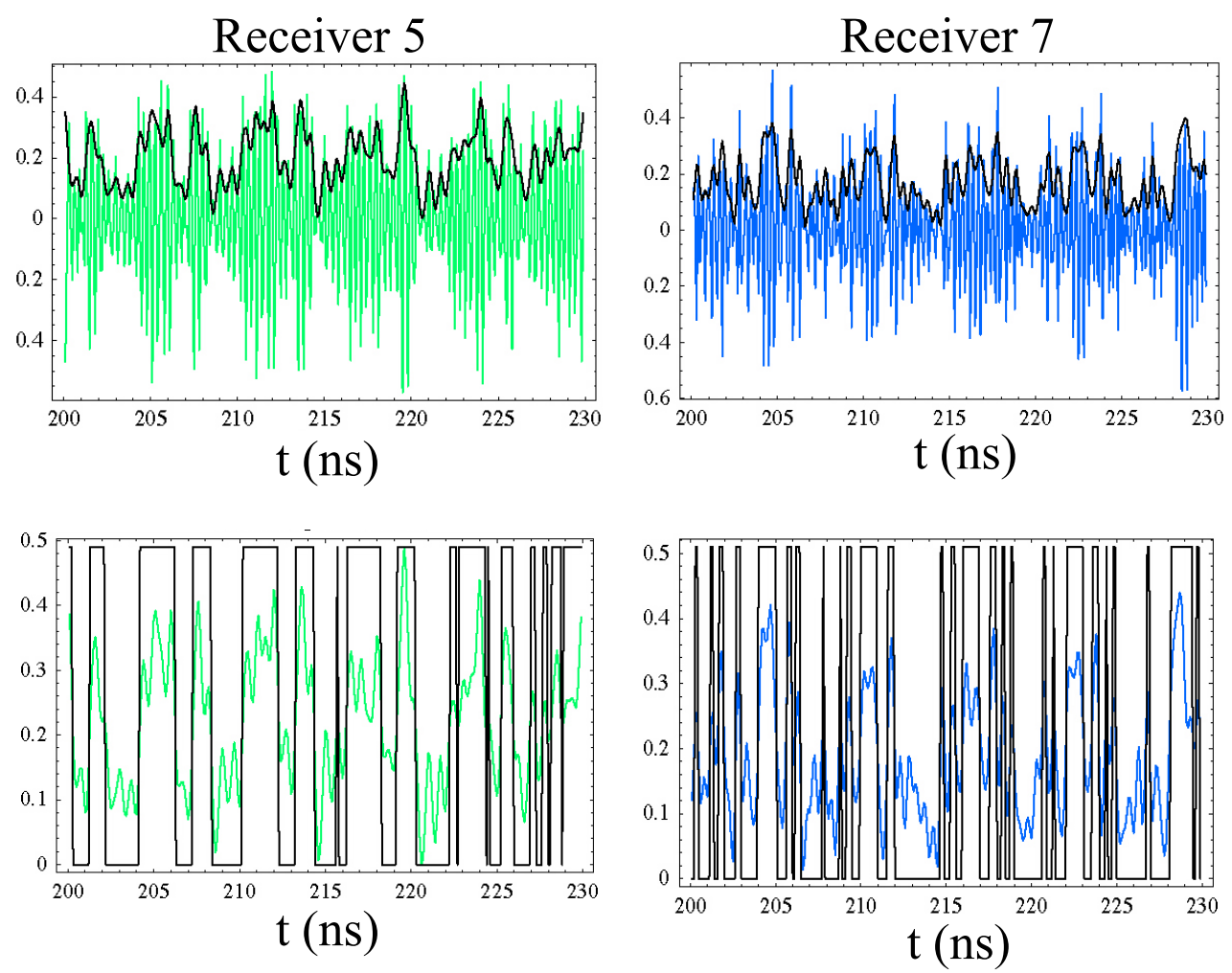

Fig. 16 Raw received signal at receivers 5 and 7 in Fig. 12 for the transmitted pulse signal of Fig. 6 on a $5 \mathrm{GHz}$ carrier. The detected signal is shown as a black line in the top figures and together with the threshold output at the bottom.
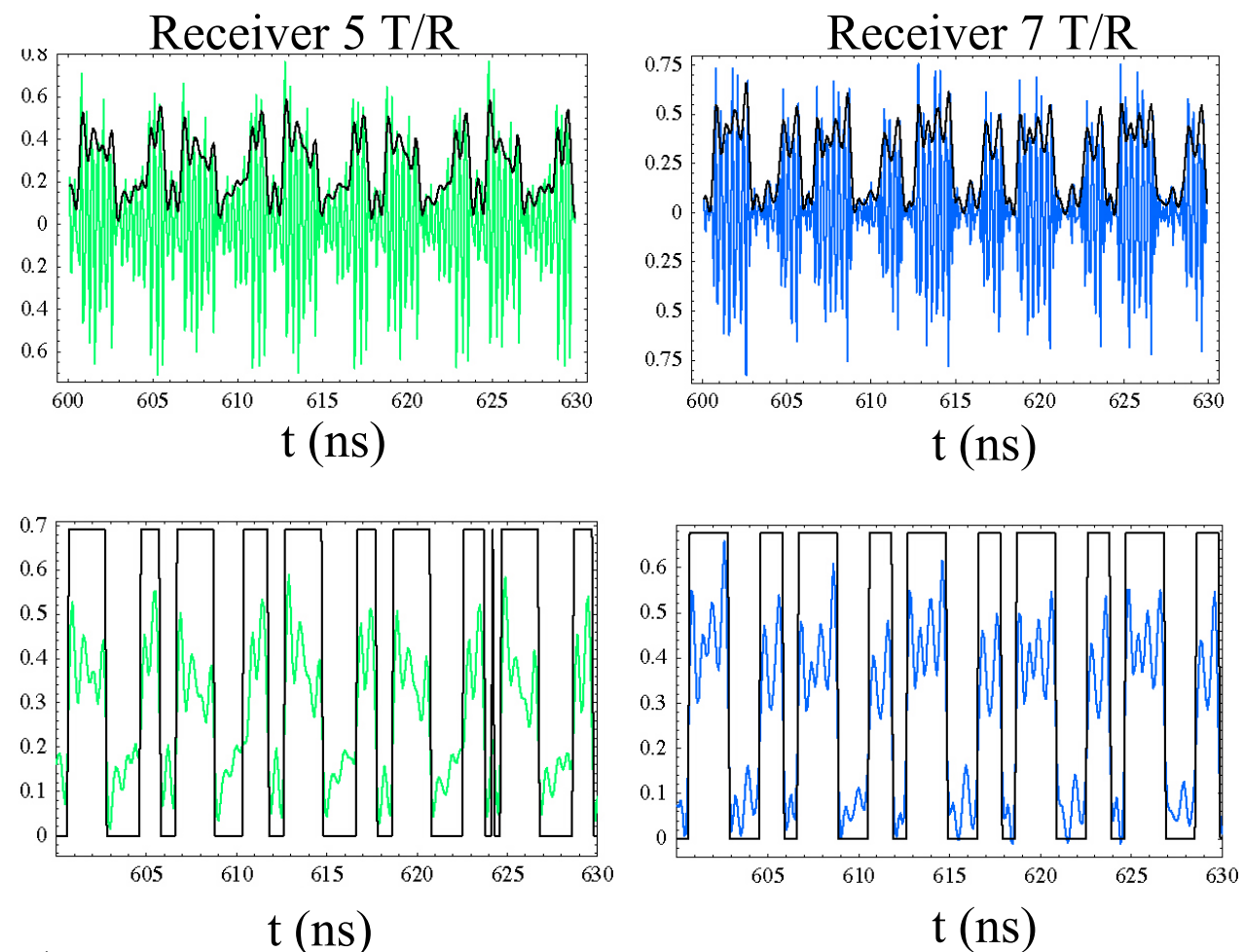

Fig. 17 Result of $\mathrm{T} / \mathrm{R}$ processing of the received signals shown in Fig. 15 for receivers 5 and 7 . The threshold for the bottom plots was set at 0.3 of the maximum of the detected signal. 


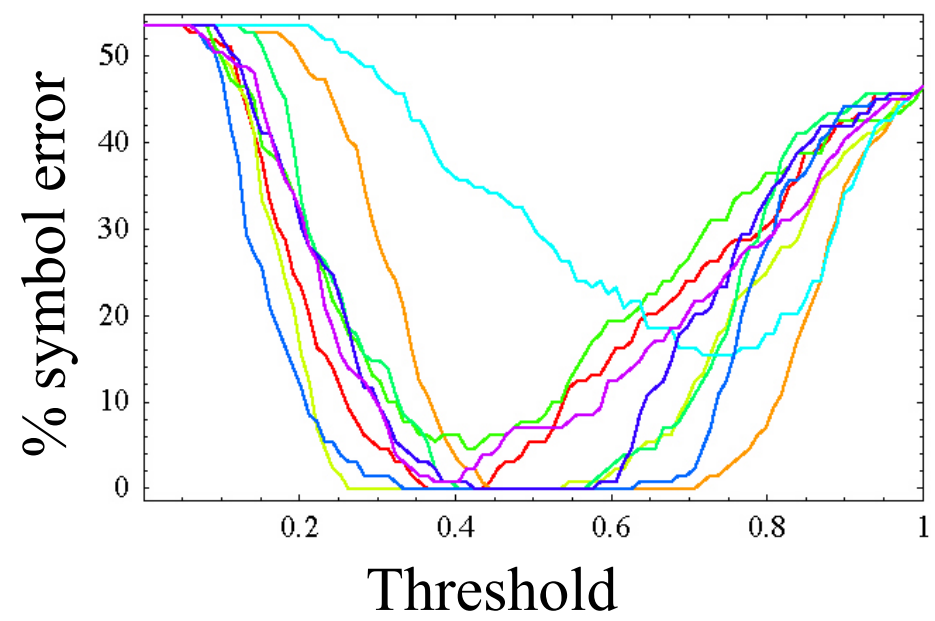

Fig. 18 Symbol error versus threshold level for the T/R signals from the 9 receivers in the cavity of Fig. 14.
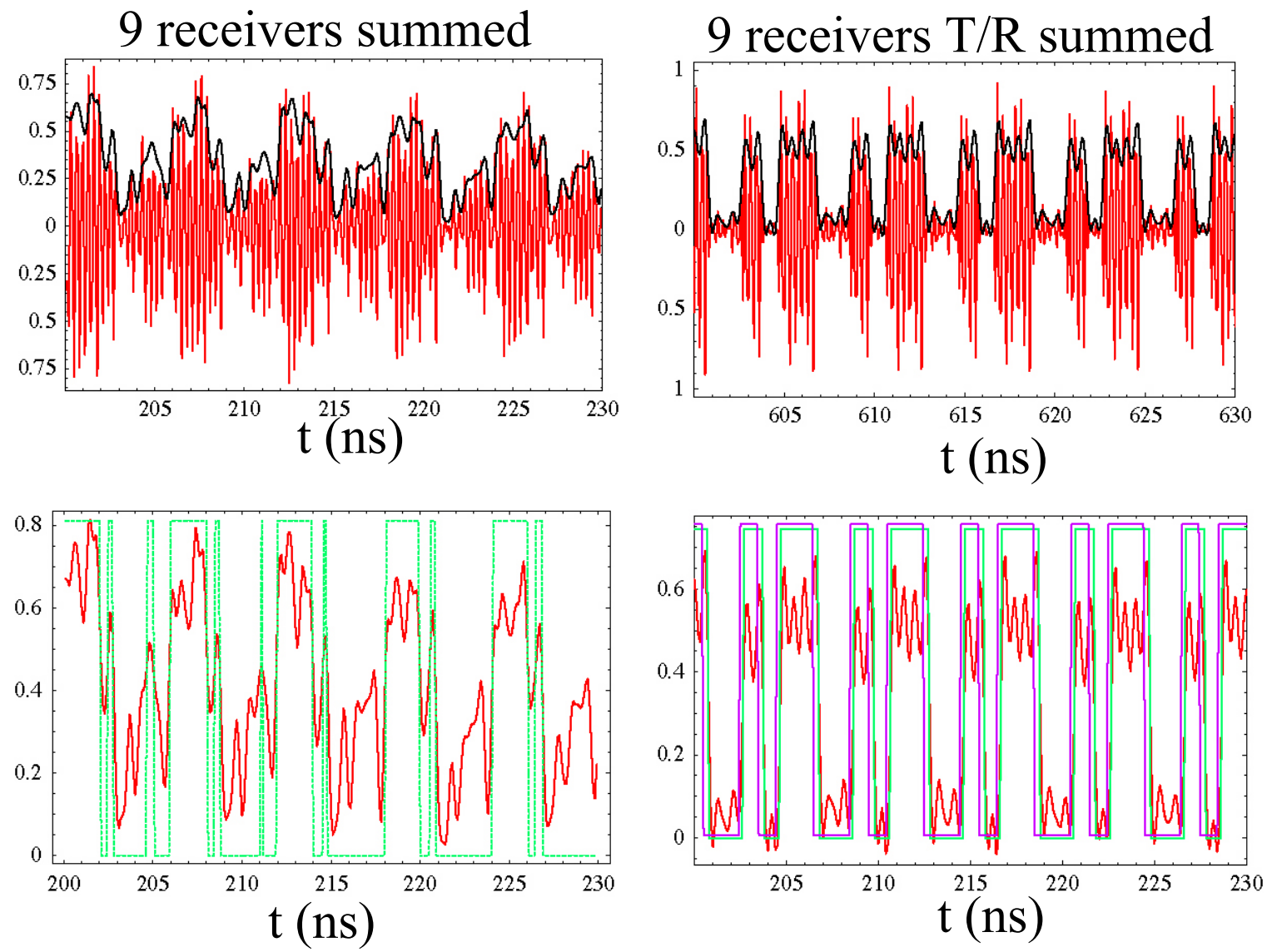

Fig. 19 Received signal from a sum of the 9 array elements in Fig. 14 and from a sum of the 9 T/R signals. Combining the $9 \mathrm{~T} / \mathrm{R}$ signals results in an error free output over a wide threshold range. 

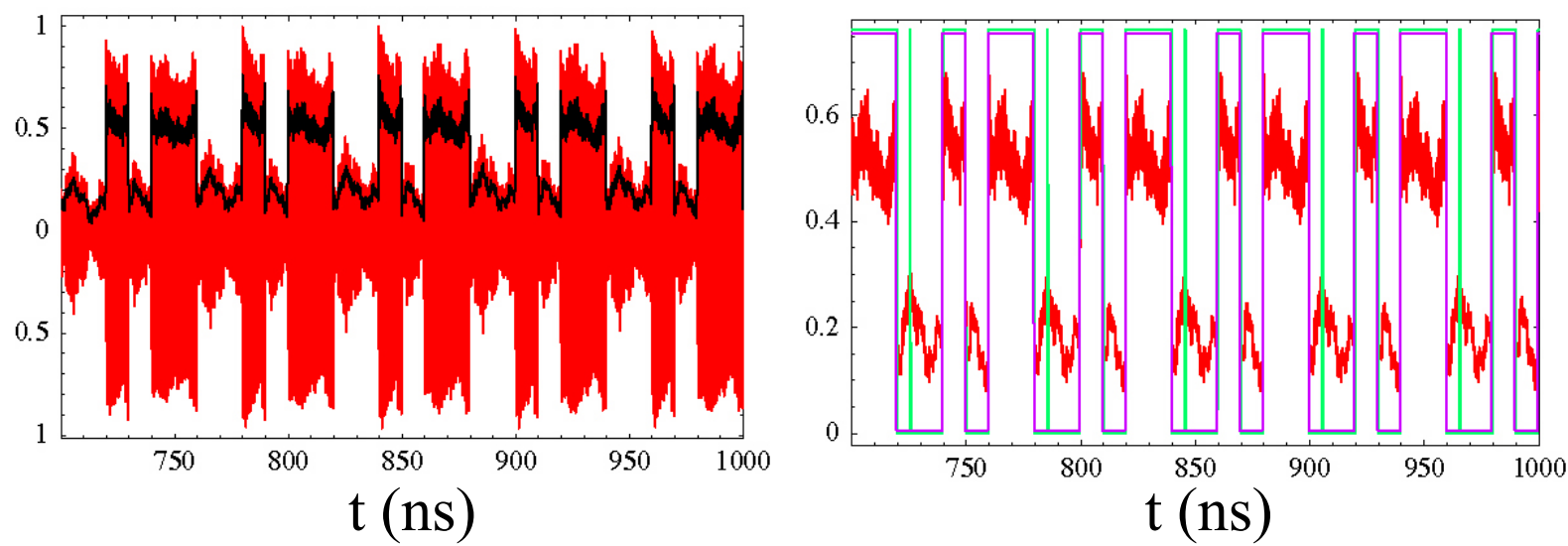

Fig. 20 Sum of the $9 \mathrm{~T} / \mathrm{R}$ signals for a $10 \mathrm{~ns}$ basic pulse width in the cavity of Fig. 14, showing the T/R output and detected signals (left) and detected and threshold output (right). The wider pulse, narrower band signal results in growth of the "noise" level from supperposition of the tails of the autocorrelation $C_{g g}(t)$.

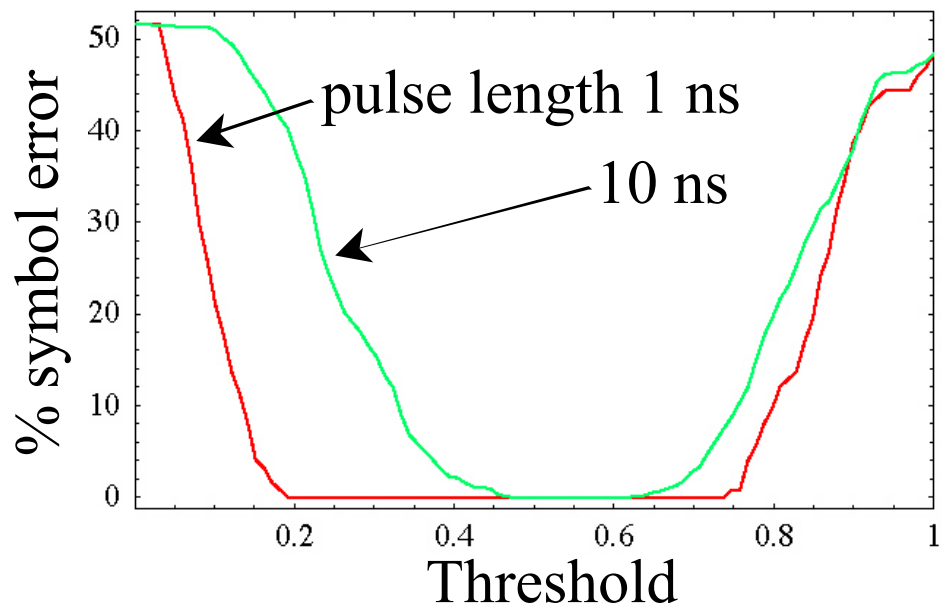

Fig. 21 Symbol error versus threshold level for the sum of 9 T/R signals of the receiver array in Fig. 14. Curves for $1 \mathrm{~ns}$ and $10 \mathrm{~ns}$ pulse width are compared. 


$$
\mid
$$

